3740.222-EN-97

June 9, 1987

\title{
Summary of ANSYS and Strain Gauge Results for the EC Calorimeter OH and MH Modules
}

Bob Wands, Kay Weber, Joe Zurawski

\section{Introduction and Background}

The $\mathrm{OH}$ and $\mathrm{MH}$ modules of the EC calorimeter consist essentially of metal boxes containing calorimetry plates. These plates can contribute to the module behaviour only in compression, with this effect being enhanced if the plates are compressively preloaded against the skin of the box prior to assembly.

The simplest approach to the analysis of these modules is beam theory. It would be expected that this would result in a crude approximation to deflection due to the small length to depth ratio of the module, which would produce shear deflections which are a large percentage of the total deflection. The question of the appropriate moment of inertia is also important, since this depends on the extent to which the internal plates contribute. However, beam theory could be successfully used in the design of the modules from the standpoint of strength in a cantilevered position. This is because it is clear that complete buckling of the compressive skin of the box is impossible due to internal plate participation, and it can be shown that a beam theory approach based on the moment of inertia of the skin only is always conservative. However, the more complex loading that results from the assembled condition is not readily modeled by this approach.

The problem of behaviour in the assembled position might be approached with an energy method. These methods use closed form approximations for the strain energy density in a structure in terms of known loads, redundant reactions, and deflections, then explicit integrations over the structure to find the total strain energy, 
followed by minimization of the resulting expressions to find various unknowns. The chief difficulty with this approach is that the strain energy expressions are generally based on simple theory (beam theory) in order to be integrable, and so carry all of the approximations of that theory. The ring resulting from the assembly of modules is not a good approximation to a thin, continuous ring, and would not therefore be expected to be modeled well by an energy method which applies beam theory to find strain energies.

The finite element method can also be applied in the analysis of these modules. It's advantages are

1. The structural components can be modeled with less simplification than beam theory allows. The angled faces of the $\mathrm{OH}$ modules can be represented exactly, and the shear deflections inherent in short, deep beams will be a natural part of the solution.

2. The finite element model can be subjected to any number of realistic loadings.

3. With proper mesh density relevant stresses can be extracted.

The disadvantages of the method are that exact modeling of the internal plates is difficult, time consuming, and computationally expensive. It is of interest, then, to verify how well a simple model of the structural components only (i.e., the skin, endplates, and any structural internal plates) predicts deflections and stresses which can be relied on for design purposes.

The finite element modeling of the $\mathrm{OH}$ and $\mathrm{MH}$ EC modules has been under constant review since the technique was first applied to these structures. Early verification attempts were based on comparison of finite element deflection predictions with measured module deflections. These 
comparisons were not entirely successful, due primarily, in the author's opinion, to the difficulty of measuring the actual module deflections with acceptable accuracy. It was proposed in October, 1986, that verification be based on stress, rather than deflection. The purpose of this report is to summarize the results of four experiments which were conducted to determine the accuracy with which ANSYS finite element models could predict the stresses in the $\mathrm{OH}$ and $\mathrm{MH}$ EC modules as measured by strain gauges.

\section{OH Cantilever Study (Nov., 1986)}

This study involved the strain gauging of an $\mathrm{OH}$ module prototype. This module had a skin thickness of $1 / 16$ th inch, and the skin did not have any holes (holes were included in later prototypes to enhance cooldown). The module was supported by one end, as will be done during installation. Strain gauge rosettes (a small assembly of three gauges which can determine principal stresses) were applied to the module prior to lifting.

An ANSYS model was generated with plate/shell elements, and constraints were applied which were thought to approximate those of the actual module. The module weight was applied with nodal forces along the two bottom edges of the module, to simulate the internal plate contact with the skin.

Figure 1 shows the strain gauge locations and the ANSYS/strain gauge results. The stresses are compared on the basis of stress intensity, which is the largest algebraic difference between principal stresses at a point. (Stress intensity is the basis of the ASME Boiler and Pressure Vessel Code, Sect. III and Sect. VIII, rules for design by analysis).

Location 1 is far from discontinuities, and the best agreement with ANSYS results would be expected there. The ratio of ANSYS to strain gauge stress intensity is 0.78 at this location, which is better agreement than at Location 2. However, of the three comparisons with actual prototype measurements presented here, this is the only instance of 
ANSYS underpredicting the measured stress. Possible reasons for this may include the relative inexperience of the analysts and experimenters in the application of the strain gauge and ANSYS techniques to these modules. It should still be noted, however, that if the ANSYS calculated stress were compared with an allowable stress which represents a safety factor of 1.5 , then the effective safety factor would still be 1.2 .

\section{MH Cantilever Study (Jan., 1987)}

This study involved a prototype of the MH module. This module used 1/16th skins on both the coarse and fine hadronic portions, and the skin was perforated with holes. Strain gauges were applied and the module was lifted by one end in the same manner as the $O H$ module.

A two step finite element procedure was used. The first step was the generation of a relatively coarse mesh (Fig. 2). This mesh did not explicitly include the effects of the holes, but approximated their effect on the module stiffness by using a reduced Young's modulus for the skin material. The results of this mesh were then used to provide displacement constraints for a refined model of the module in the vacinity of the strain gauge (Fig. 3). The detailed submodel included holes and the real Young's modulus for the skin.

Fig. 4 shows the results of this comparison for two locations on the MH module. There was a large reduction in calculated stress at the strain gauge location produced by the detailed modeling. At both locations the tendency is for the finite element model to overestimate the stresses as measured by the strain gauges.

Two strain gauges were used at Location 1 (one on either side of the module). The two gauges failed to give consistent readings. Two possible reasons for this are faulty installation of a gauge, or uneven distribution of the internal plate weight to the side skins. In any case, agreement is quite acceptable given the high stress gradients found in the area, and the presence of holes in the region near the gauge. 


\section{OH Crushing Tests (April, 1087)}

An $\mathrm{OH}$ module prototype with $1 / 32$ nd in. skins with holes was strain gauged and subjected to a crushing load test as shown in Fig. 5 . The results of this test were compared with those of an ANSYS model of the module (Fig. 6) which, as with all models in this work, included only the skins, endplates, and internal structural plates.

The best agreement was found where expected, at Location 5 , shown in Fig. 7. This location is far from discontinuities such as edges and holes, and gives excellent agreement throughout the elastic range of the material. Good agreement in the elastic range is also found at Location 9 (Fig. 8). This gauge is also far from discontinuities.

The ANSYS stresses at the remaining three locations (Figs. 9-11) are at least a factor of two larger than the measured stresses. These locations are all on the side skin, which is in a complex stress state which includes the possibility of local buckling or wrinkling, and good agreement should not be expected. It should be noted that ANSYS consistently overestimates the stress at these locations, however, and a design based on the ANSYS values for this loading would be conservative.

\section{MH Skin-Only Load Test (May, 1987)}

All comparisons up to this point had been between an actual module prototype and an approximate ANSYS model. It was decided to remove the variability caused by the internal calorimeter plates, and conduct a load test of an MH skin-only module section. This could then be directly compared with the results from an ANSYS skin-only model, and the accuracy obtainable in the absence of internal plate complications could be assessed.

The module was supported and loaded as shown in Fig. 12. The stresses at various locations were measured, as well as the tip deflection. Ten strain gauge rosettes were mounted. Gauges 4 and 10 were on the compressive side of the module, and were mounted at identical distances from the supported end. Gauge 4 was mounted on the outside of the 
skin, and gauge 10 was mounted on the inside. It was hoped that this arrangement might pick up buckling tendencies in the compressivly loaded skin.

As seen in the Figure, the tip deflection as predicted by ANSYS was $83 \%$ of the measured tip deflection, which is excellent agreement given the small numbers being measured.(It is known in the classic application of the finite element technique that the finite element model will always be stiffer than the real structure, and so will tend to underpredict deflections)

Fig. 13 shows a comparison of the strain gauge and ANSYS stress intensities for gauge 5, a location on the skin well away from discontinuities. As in the case of the prototype comparisons, the agreement is excellent. Agreement is also very good for gauges 8 and 9 (Fig. 14 and 15), which are on the tensile side of the module. (The sharp peak in the data for gauge 8 represents debonding of the strain gauge. This gauge later fell off of the module)

Fig. 16 shows the double gauge results for gauges 4 and 10. From the strain gauge plots alone it appears that buckling (represented by disimilar slope) began in earnest at a relatively low load of 4000 lbs. However, in a thin skinned box such as this, buckling of the central portion of the skin will transfer load carrying to the edges of the box. It appears that this has occured at a point load of $10000 \mathrm{lbs}$, at which point the gauges are once again exhibiting the same load/strain behaviour. The ANSYS results are plotted as a double line, representing the stress on the inside and outside of the skin. There is a very small bending component in the finite element results. The ANSYS stresses agree well for these locations.

Figs. 17-19 show the results at locations on the side skins at various distances from the supported end and upper or lower skin. The results for gauge 6 show acceptable agreement, which might be expected given the tensile stress state at this location. Agreement for gauge 2, on the compressive part of the side skin, is not as good, with ANSYS predicting approximately half of the measured stress. Gauge 3 shows the worst 
agreement, and observations during the test showed that this location began to wrinkle almost immediately upon application of the load. The poor agreement is not surprising.

It seems clear from this comparison that ANSYS can acceptably predict many of the stresses in the skin-only module. The most difficult areas to predict stresses are the compressive portions of the skin, as expected, and those areas near the support or corners of the module.

\section{Conclusion}

The three comparisons with actual module prototypes show that ANSYS can predict with good accuracy the stresses in those regions far from discontinuities where the stress gradient is low. In all regions, but particularly those of high gradient, ANSYS will tend to overestimate the stress.

The comparison with the skin-only module shows that the basic approach is sound and exhibits the behaviour expected from a finite element analysis.

Finite element analysis can clearly be a useful part of the module design process when augmented by experimental and closed-form analytical techniques. 


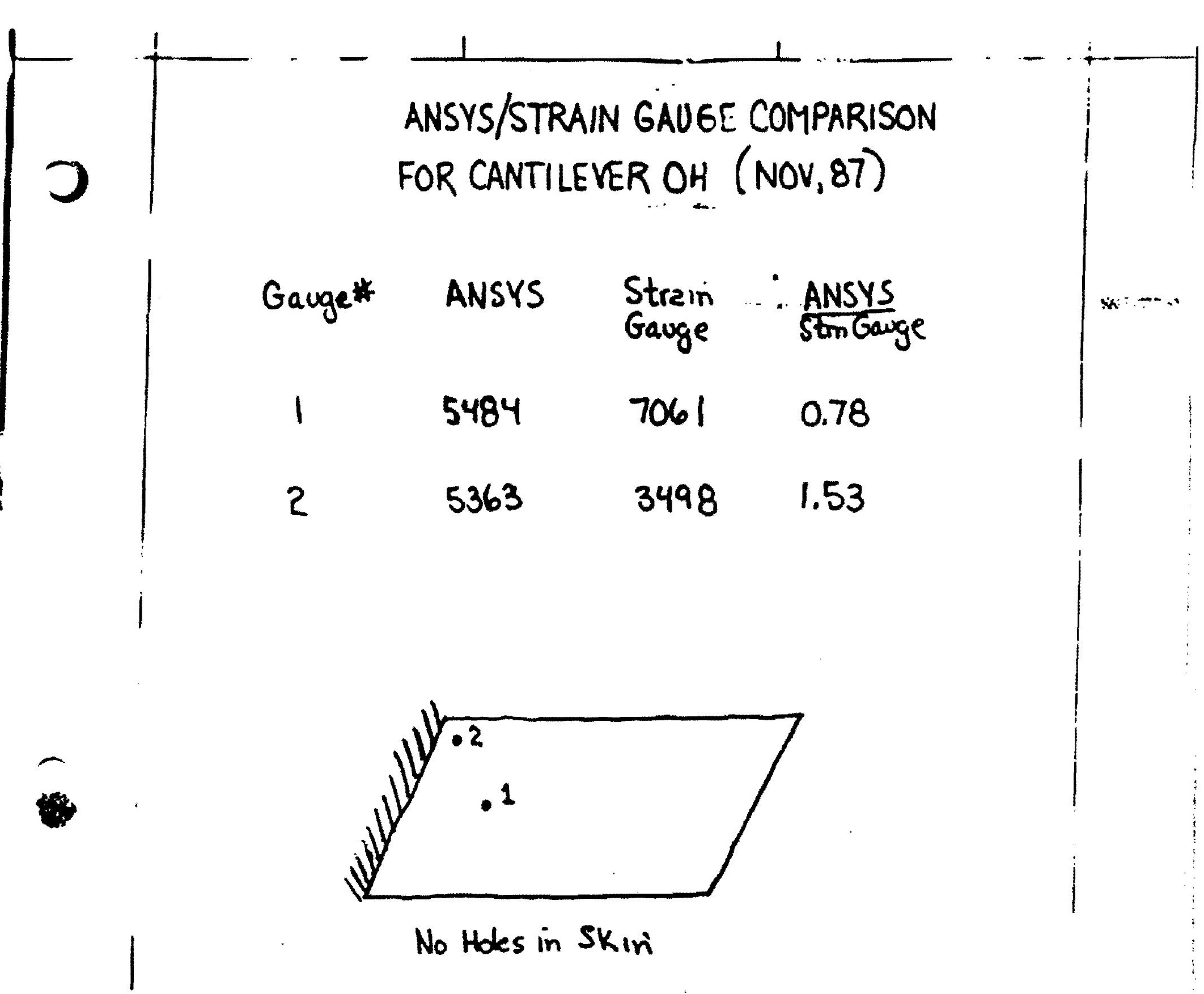

Fig. 1 


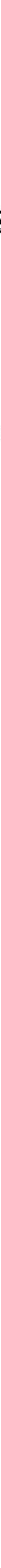




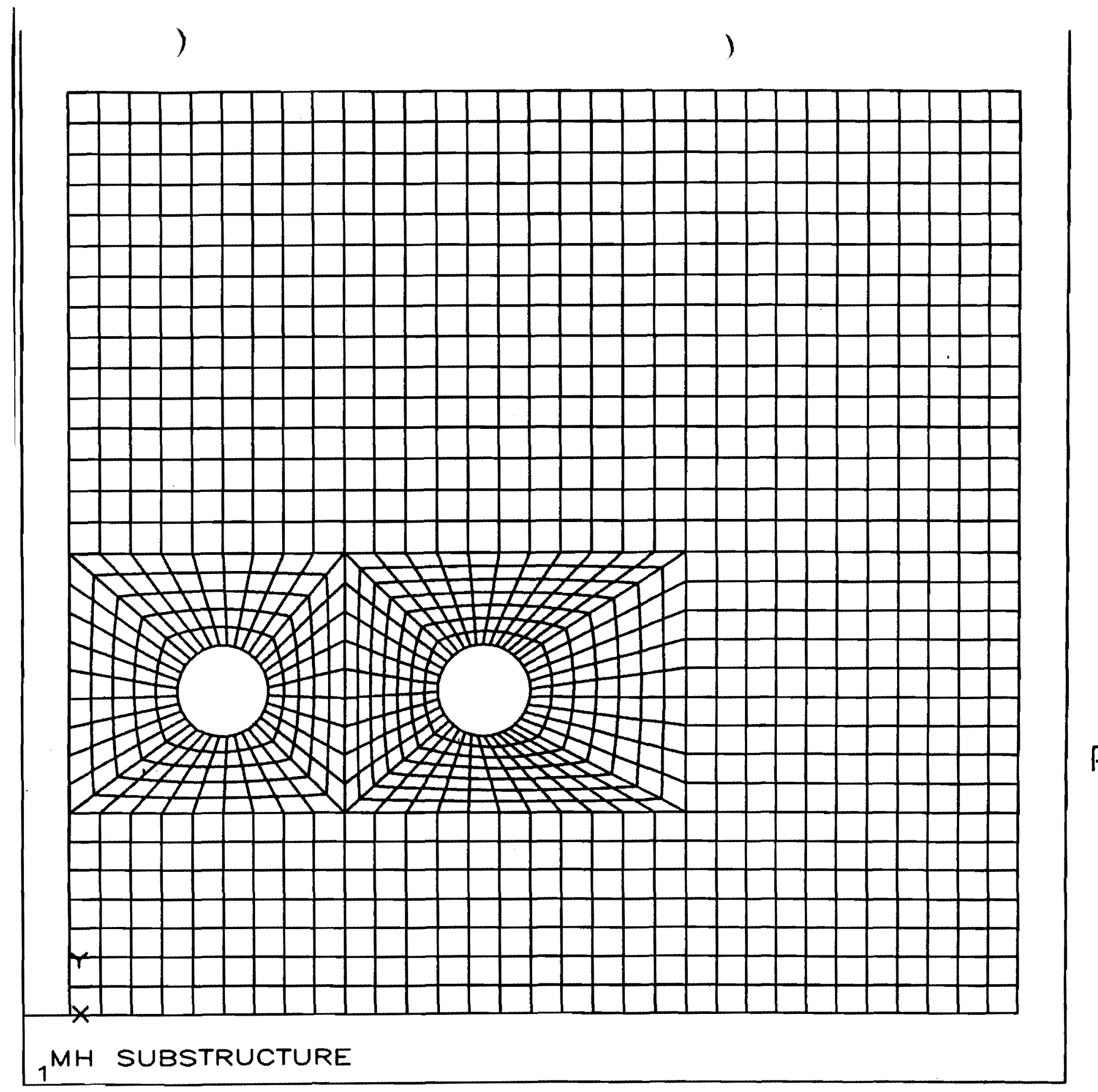

ANSIS $14.2 B$

MAR $26 \quad 1987$$$
\text { 9:33:49 }
$$$$
\text { PLOT NO. } 1
$$$$
\text { PREP 7 ELEMENTS }
$$$$
\text { ORIG SCALING }
$$$$
X V=1
$$$$
\mathrm{DIST}=4.22
$$$$
X F=-7.69
$$$$
Y F=3.67
$$$$
Z F=-3.84
$$

Fig 3. Submodel Mesh for MH Cantilever 

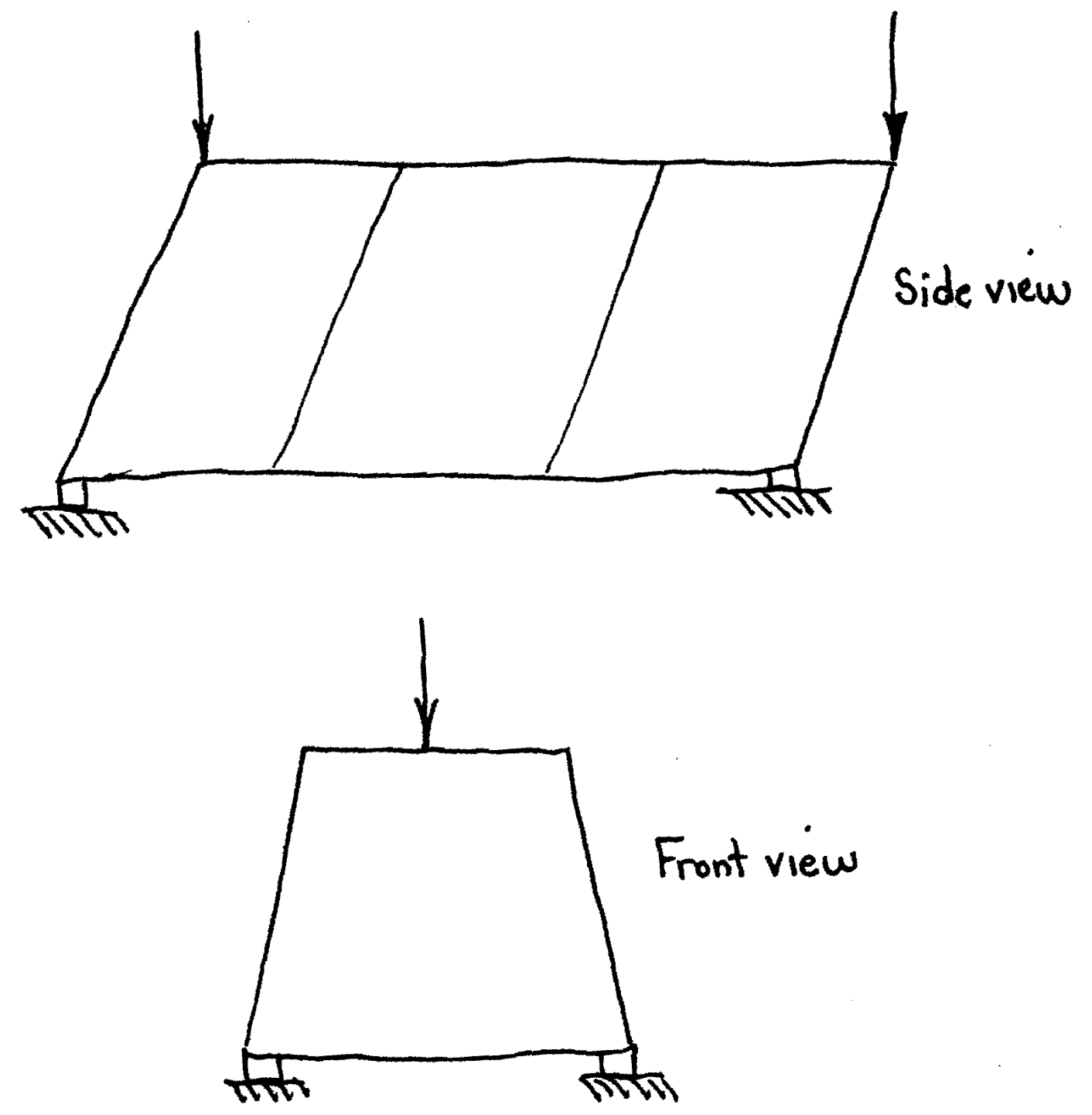

Fig 5. Loading for OH Crushing Comparison 


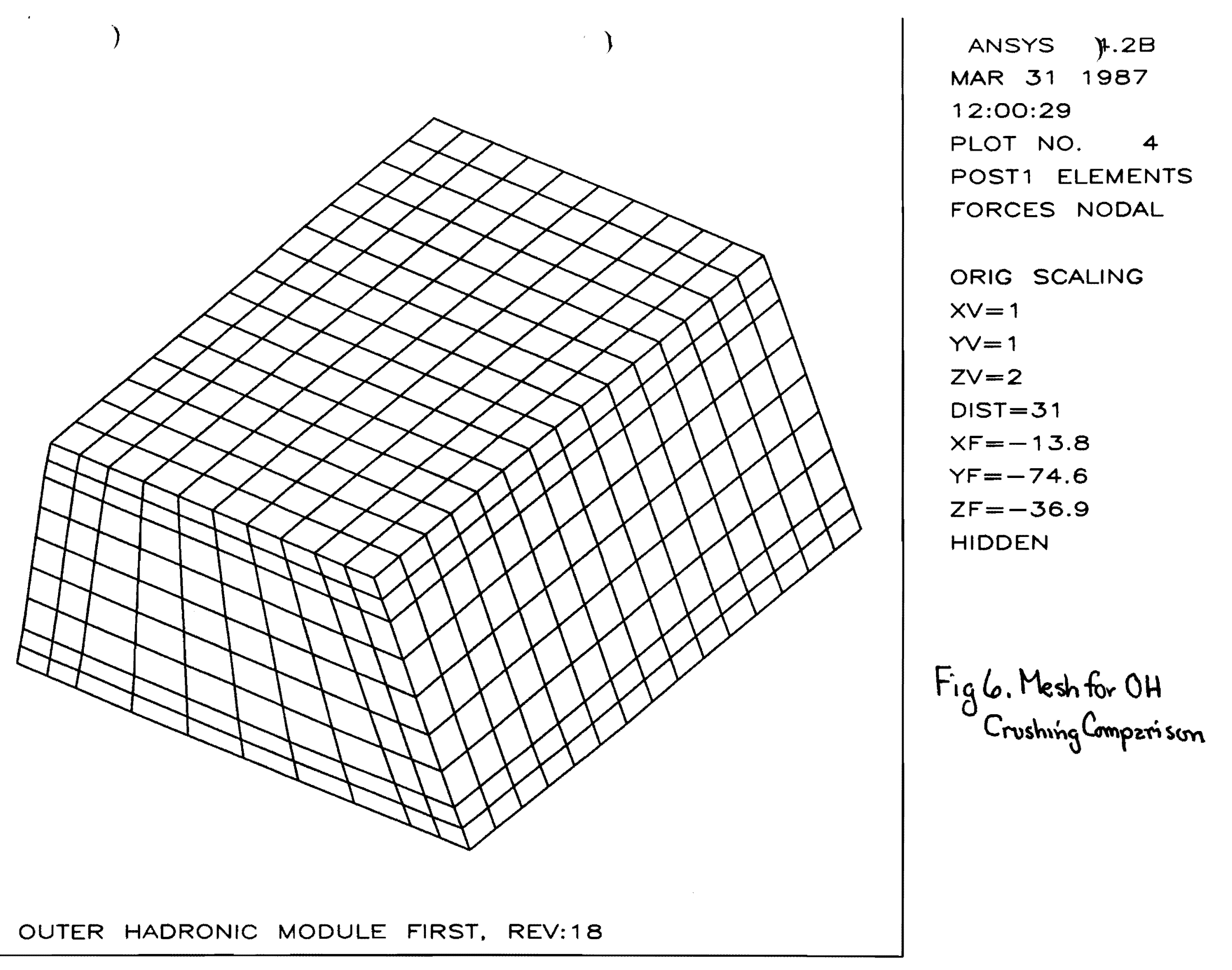




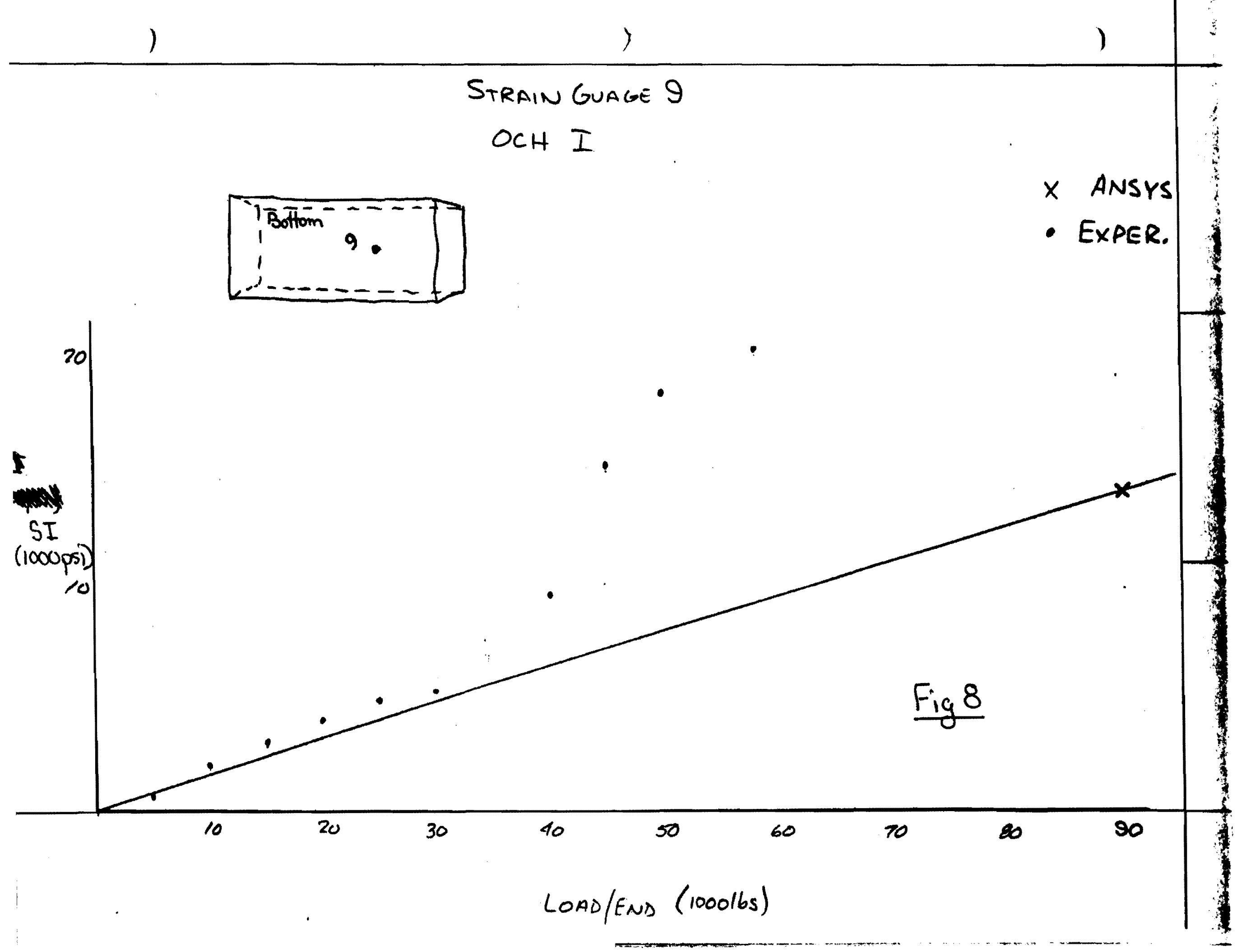




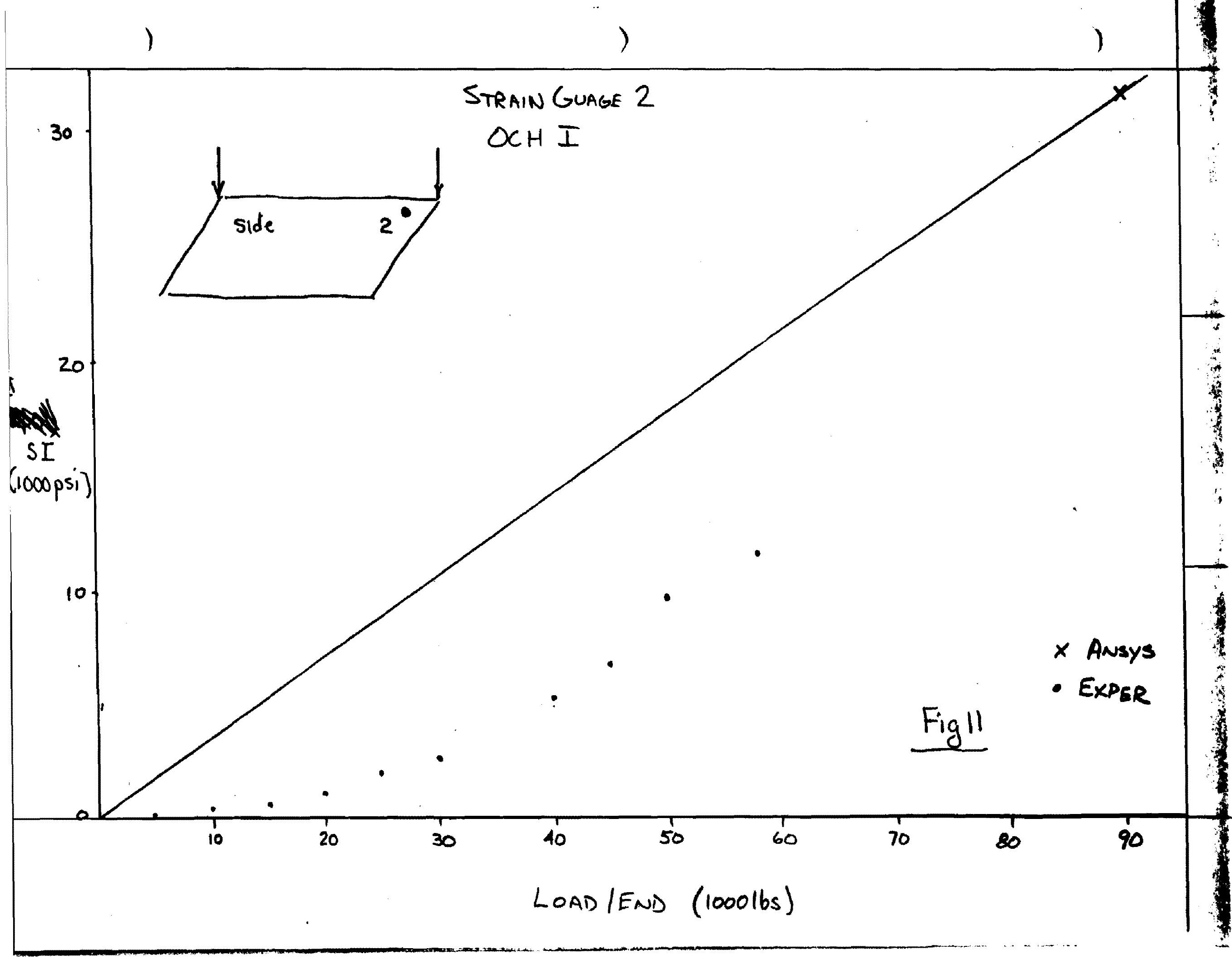




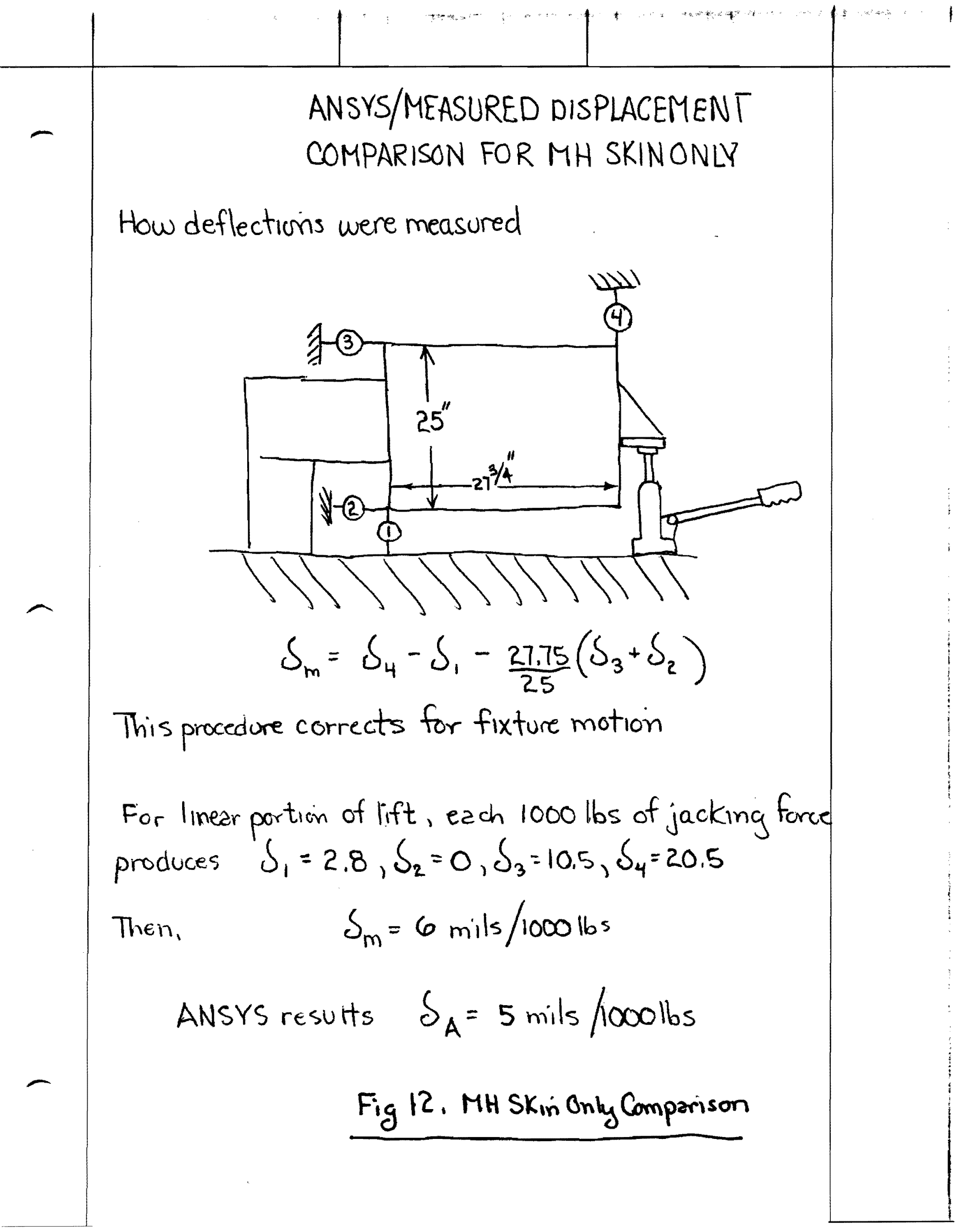




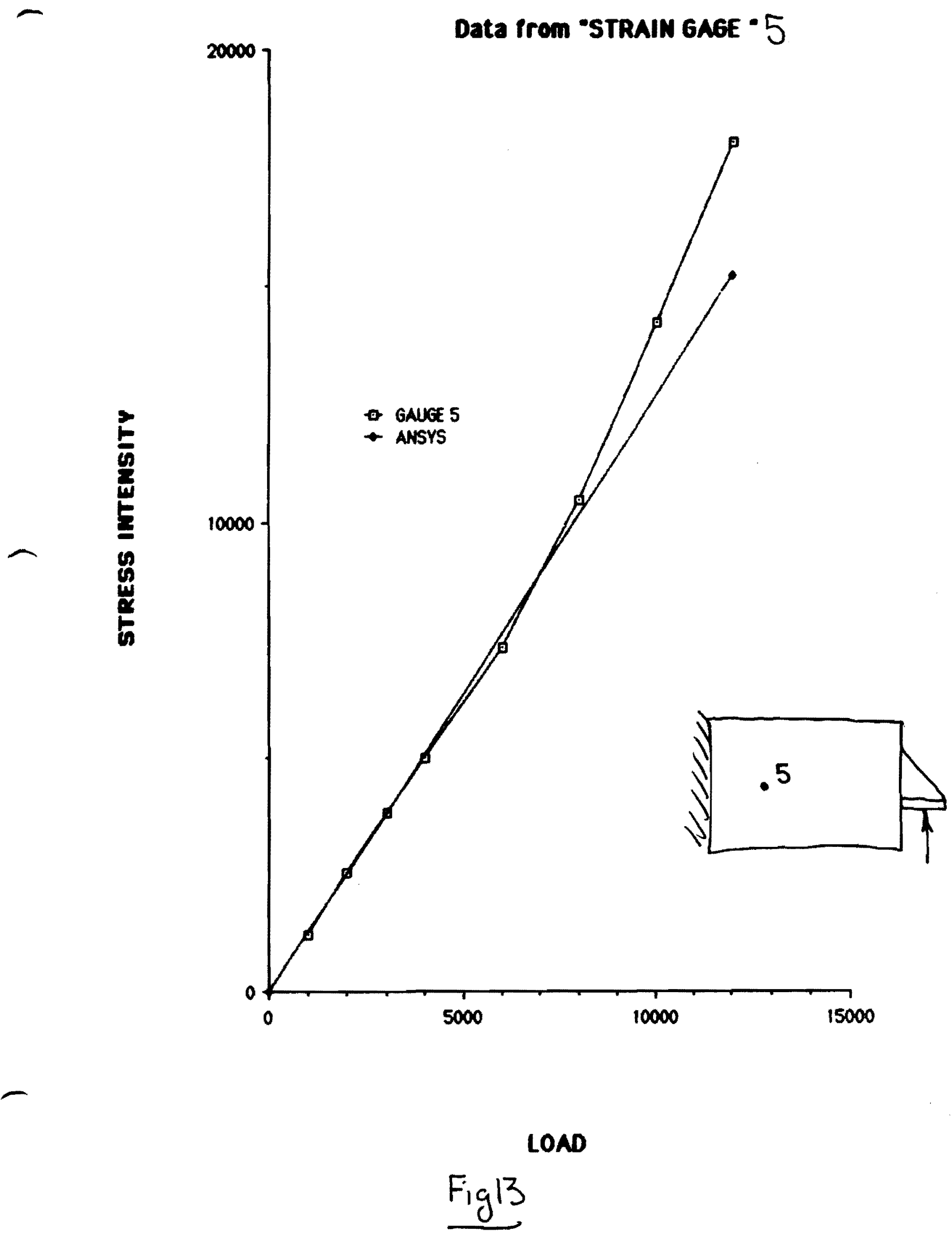


Data from "sTrain GAEE - 8
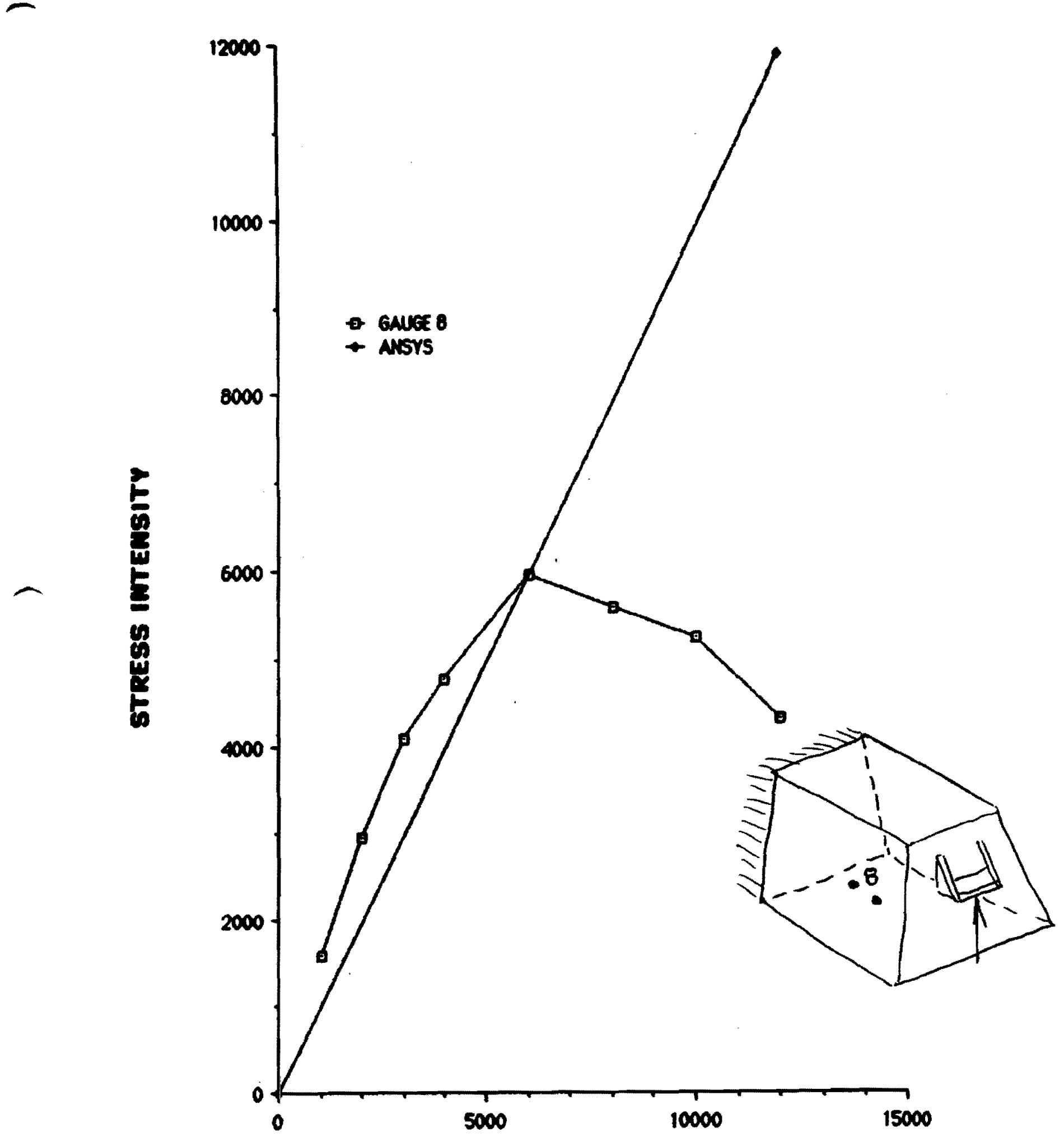

Fig 14

LOAD 


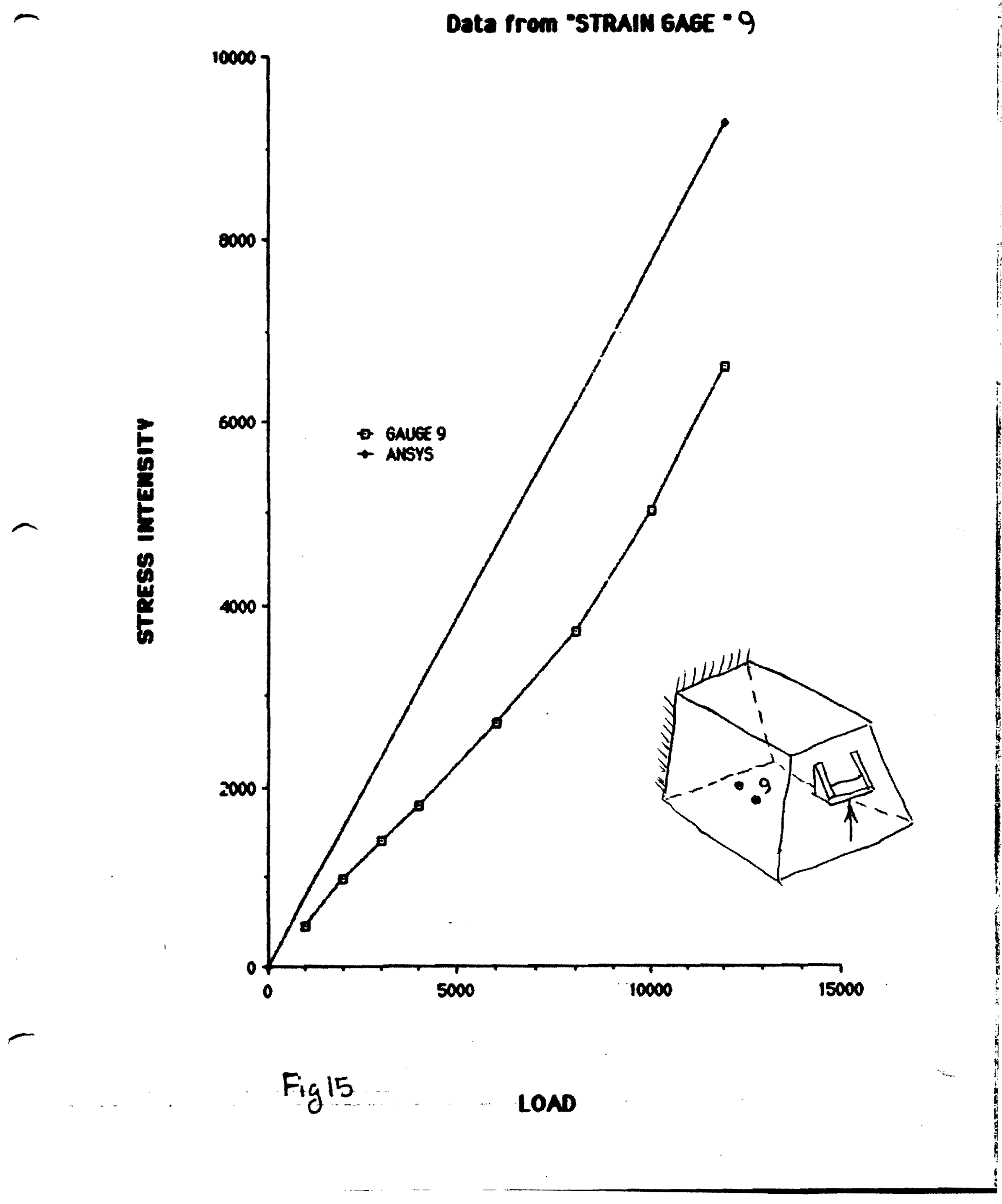




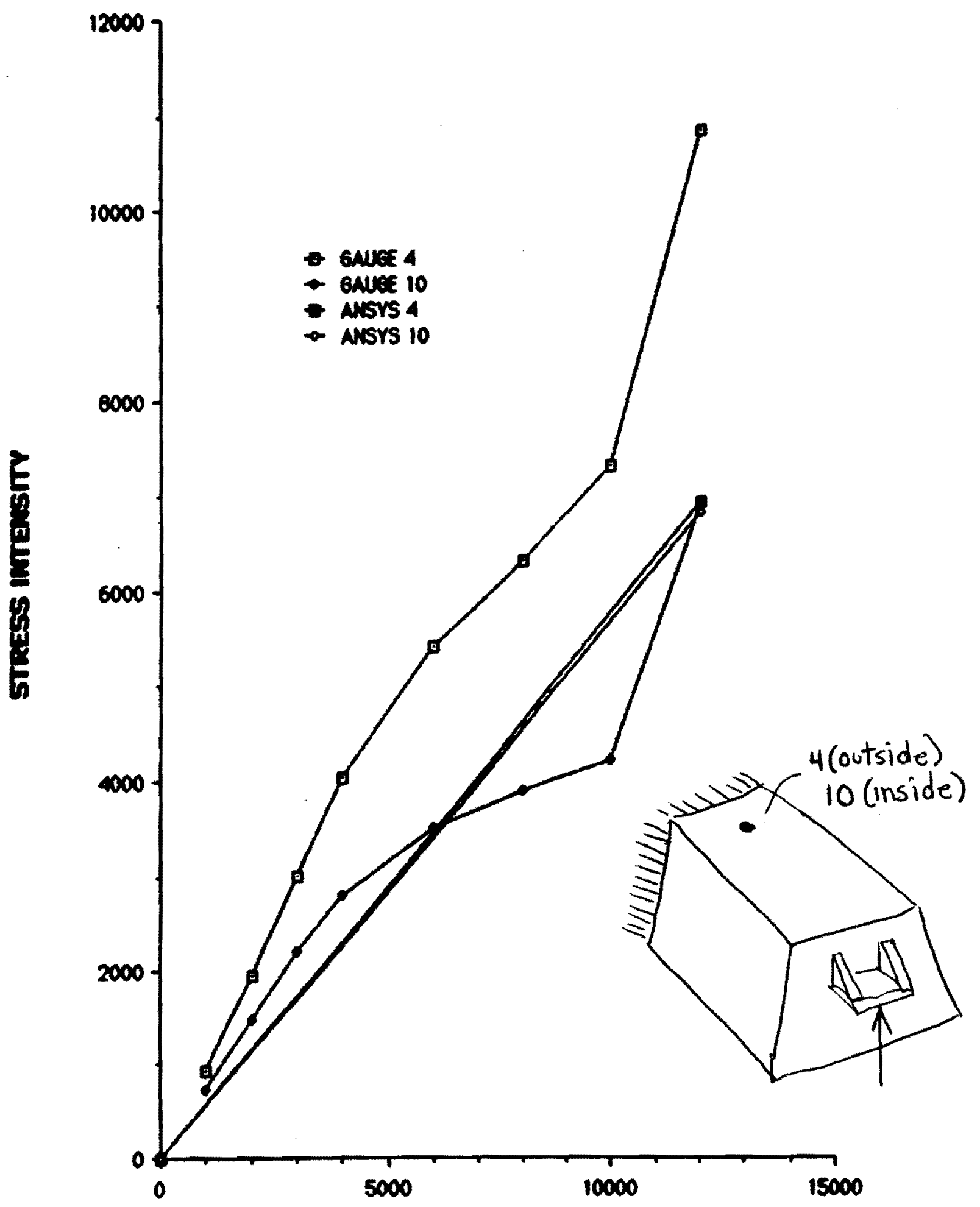

Figl6 $\quad$ LOND 


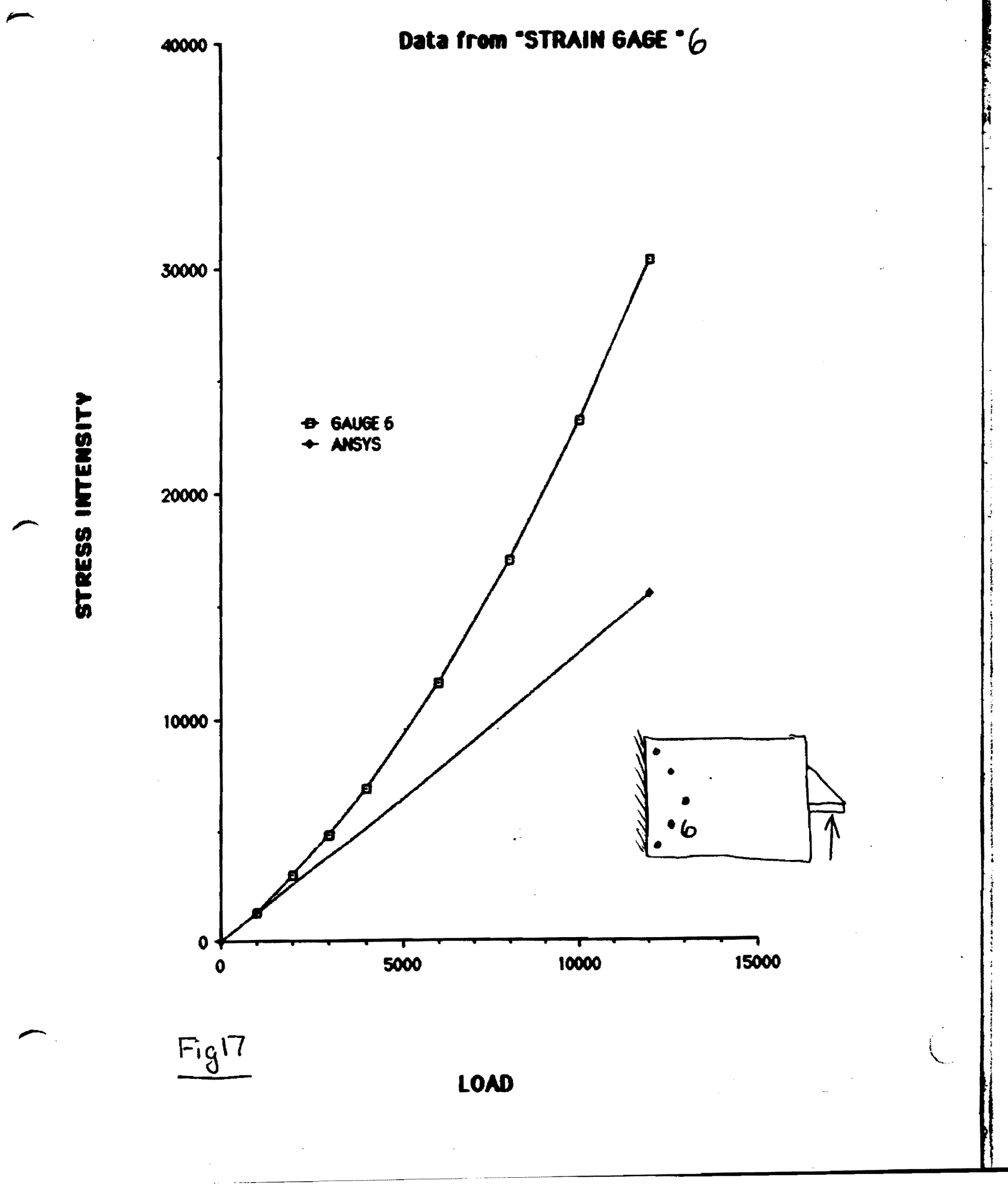




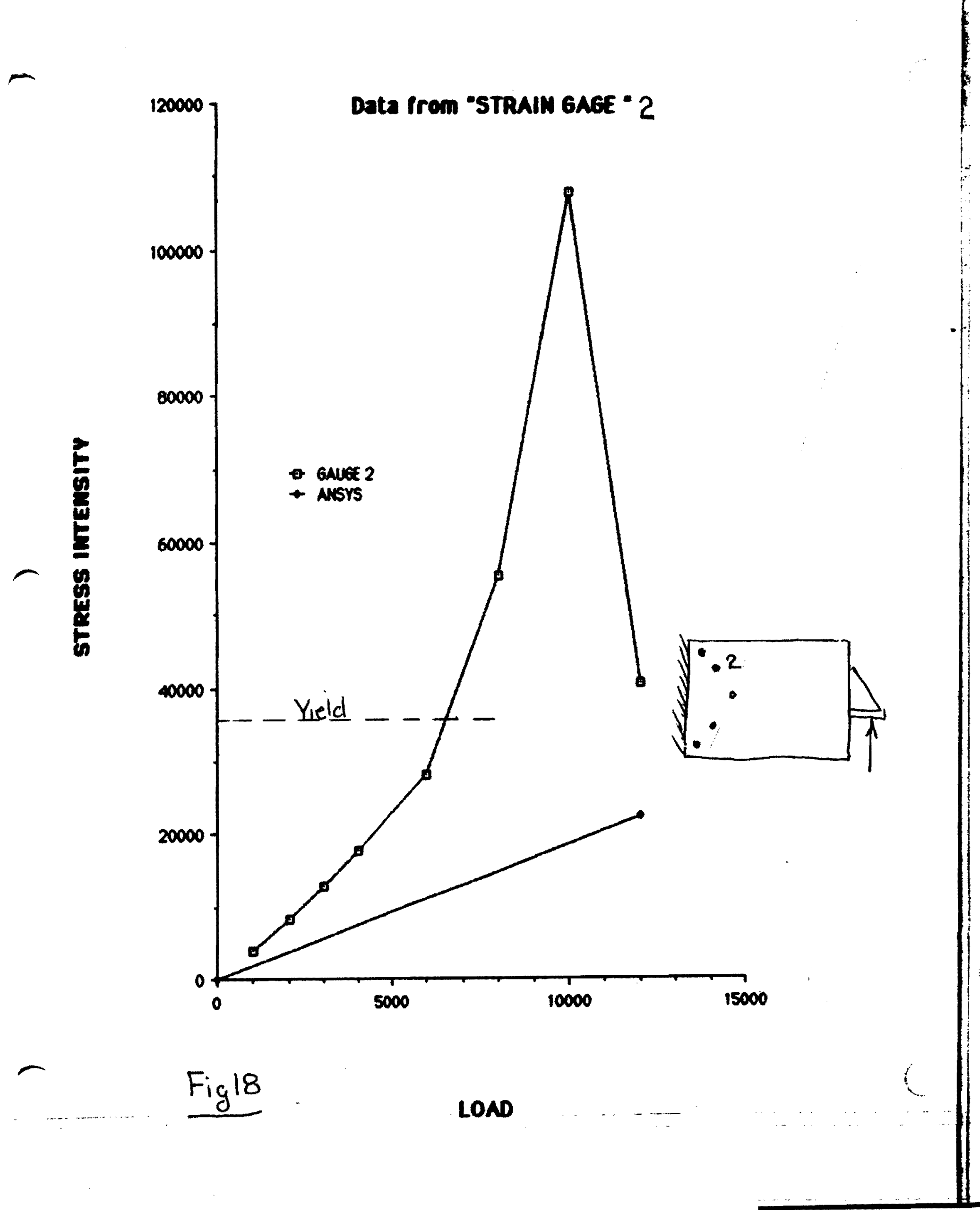




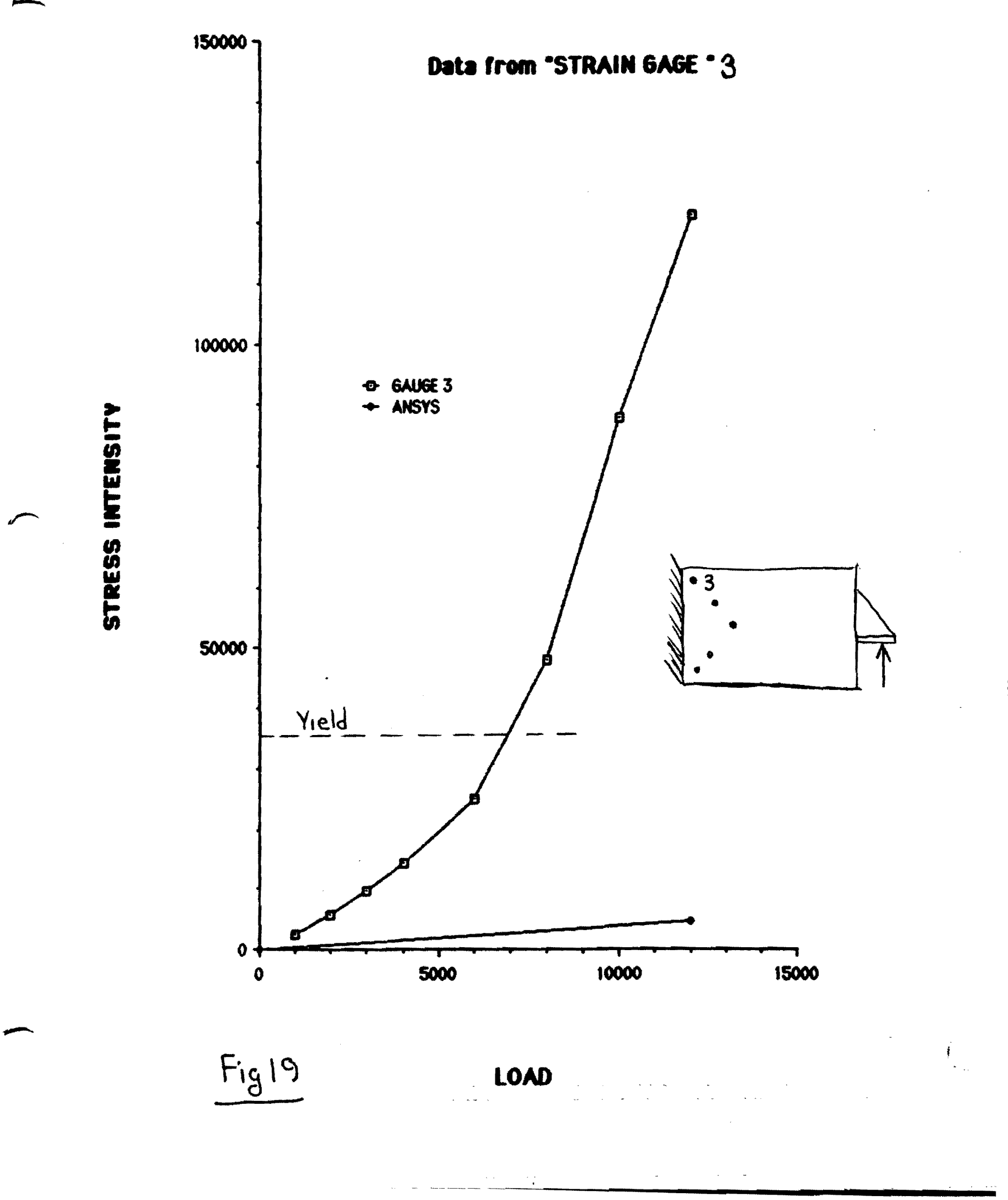

